\title{
FIRST BOUNDS ON THE HIGH-ENERGY EMISSION FROM ISOLATED WOLF-RAYET BINARY SYSTEMS
}

E. Aliu,${ }^{1}$ H. Anderhub,${ }^{2}$ L. A. Antonelli,${ }^{3}$ P. Antoranz,${ }^{4}$ M. Backes, ${ }^{5}$ C. Baixeras,${ }^{6}$ J. A. Barrio, ${ }^{4}$ H. Bartko, ${ }^{7}$ D. Bastieri, ${ }^{8}$ J. K. Becker,${ }^{5}$ W. Bednarek, ${ }^{9}$ K. Berger, ${ }^{10}$ E. Bernardini, ${ }^{11}$ C. Bigongiari, ${ }^{8}$ A. Biland, ${ }^{2}$ R. K. Bock, ${ }^{7,8}$ G. Bonnoli,${ }^{12}$ P. Bordas, ${ }^{13}$ V. Bosch-Ramon, ${ }^{13}$ T. Bretz, ${ }^{10}$ I. Britvitch, ${ }^{2}$ M. Camara, ${ }^{4}$ E. Carmona, ${ }^{7}$ A. Chilingarian, ${ }^{14}$ S. Commichau, ${ }^{2}$ J. L. Contreras, ${ }^{4}$ J. Cortina, ${ }^{1}$ M. T. Costado, ${ }^{15,16}$ S. Covino, ${ }^{3}$ V. Curtef, ${ }^{5}$ F. Dazzi, ${ }^{8}$ A. De Angelis, ${ }^{17}$ E. De Cea del Pozo, ${ }^{18}$ R. De los Reyes, ${ }^{4}$ B. De Lotto, ${ }^{17}$ M. De Maria, ${ }^{17}$ F. De Sabata, ${ }^{17}$ C. Delgado Mendez, ${ }^{15}$ A. Dominguez, ${ }^{19}$ D. Dorner,${ }^{10}$ M. Doro,${ }^{8}$ M. Errando, ${ }^{1}$ M. Fagiolini,${ }^{12}$ D. Ferenc, ${ }^{20}$ E. Fernández, ${ }^{1}$ R. Firpo, ${ }^{1}$ M. V. Fonseca, ${ }^{4}$ L. Font,${ }^{6}$ N. Galante, ${ }^{7}$ R. J. García López, ${ }^{15,16}$ M. Garczarczyk, ${ }^{7}$ M. Gaug, ${ }^{15}$ F. Goebel, ${ }^{7}$ M. Hayashida, ${ }^{7}$ A. Herrero, ${ }^{15,16}$ D. Höhne, ${ }^{10}$ J. Hose, ${ }^{7}$ C. C. Hsu, ${ }^{7}$ S. Huber, ${ }^{10}$ T. Jogler, ${ }^{7}$ D. Kranich, ${ }^{2}$ A. La Barbera, ${ }^{3}$ A. Laille, ${ }^{20}$ E. Leonardo, ${ }^{12}$ E. Lindfors,${ }^{21}$ S. Lombardi, ${ }^{8}$ F. Longo, ${ }^{17}$ M. López, ${ }^{8}$ E. Lorenz, ${ }^{2,7}$ P. Majumdar, ${ }^{11}$ G. Maneva,${ }^{22}$ N. Mankuzhiyil,${ }^{17}$ K. Mannheim, ${ }^{10}$ L. Maraschi, ${ }^{3}$ M. Mariotti, ${ }^{8}$ M. Martínez, ${ }^{1}$ D. Mazin, ${ }^{1}$ M. Meucci, ${ }^{12}$ M. Meyer, ${ }^{10}$ J. M. Miranda, ${ }^{4}$ R. Mirzoyan, ${ }^{7}$ M. Moles,${ }^{19}$ A. Moralejo, ${ }^{1}$ D. Nieto, ${ }^{4}$ K. Nilsson, ${ }^{21}$ J. Ninkovic ${ }^{7}$ E. Oña-Wilhelmi, ${ }^{1,27}$ N. Otte,${ }^{7,23,28}$ I. Oya ${ }^{4}$ R. Paoletti, ${ }^{12}$ J. M. Paredes, ${ }^{13}$ M. Pasanen, ${ }^{21}$ D. Pascoli ${ }^{8}$ F. Pauss, ${ }^{2}$ R. G. Pegna, ${ }^{12}$ M. A. Perez-Torres, ${ }^{19}$ M. Persic,${ }^{17,24}$ L. Peruzzo, ${ }^{8}$ A. Piccioli, ${ }^{12}$ F. Prada, ${ }^{19}$ E. Prandini, ${ }^{8}$ N. Puchades, ${ }^{1}$ A. Raymers, ${ }^{14}$ W. Rhode, ${ }^{5}$ M. Ribó, ${ }^{13}$ J. Rico, ${ }^{25,1,29}$ M. Rissi, ${ }^{2}$ A. Robert, ${ }^{6}$ S. Rügamer, ${ }^{10}$ A. Saggion, ${ }^{8}$ T. Y. Saito, ${ }^{7}$ M. Salvati, ${ }^{3}$ M. Sanchez-Conde, ${ }^{19}$ P. Sartori, ${ }^{8}$ K. Satalecka, ${ }^{11}$ V. Scalzotto, ${ }^{8}$ V. Scapin, ${ }^{17}$ T. Schweizer, ${ }^{7}$ M. Shayduk, ${ }^{7}$ K. Shinozaki, ${ }^{7}$ S. N. Shore, ${ }^{26}$ N. Sidro, ${ }^{1}$ A. Sierpowska-Bartosik, ${ }^{18}$ A. Sillanpää, ${ }^{21}$ D. Sobczynska, ${ }^{9}$ F. Spanier, ${ }^{10}$ A. Stamerra, ${ }^{12}$ L. S. Stark, ${ }^{2}$ L. Takalo, ${ }^{21}$ F. Tavecchio, ${ }^{3}$ P. Temnikov, ${ }^{22}$ D. Tescaro, ${ }^{1}$ M. Teshima, ${ }^{7}$ M. Tluczykont, ${ }^{11}$ D. F. Torres,${ }^{25,18,29}$ N. Turini, ${ }^{12}$ H. Vankov,${ }^{22}$ A. Venturini, ${ }^{8}$ V. Vitale, ${ }^{17}$ R. M. Wagner, ${ }^{7}$ W. Wittek, ${ }^{7}$ V. ZABAlZA, ${ }^{13}$ F. ZANDANEL, ${ }^{19}$ R. ZANin, ${ }^{1}$ AND J. ZAPATERO ${ }^{6}$

Received 2008 July 22; accepted 2008 August 13; published 2008 September 9

\section{ABSTRACT}

High-energy gamma-ray emission is theoretically expected to arise in tight binary star systems (with high mass loss and high-velocity winds), although the evidence of this relationship has proven to be elusive so far. Here we present the first bounds on this putative emission from isolated Wolf-Rayet (WR) star binaries, WR 147 and WR 146, obtained from observations with the MAGIC telescope.

Subject heading: gamma rays: observations

\section{INTRODUCTION}

WR stars represent an evolved stage of hot $\left(T_{\text {eff }}>20,000\right.$ $\mathrm{K})$, massive $\left(M_{\text {ZAMS }}>25 M_{\odot}\right)$ stars, and display some of the

${ }^{1}$ IFAE, Edifici Cn., Campus UAB, E-08193 Bellaterra, Spain.

${ }^{2}$ ETH Zurich, CH-8093 Zurich, Switzerland.

${ }^{3}$ INAF National Institute for Astrophysics, I-00136 Rome, Italy.

${ }^{4}$ Universidad Complutense, E-28040 Madrid, Spain.

5 Technische Universität Dortmund, D-44221 Dortmund, Germany.

${ }^{6}$ Universitat Autònoma de Barcelona, E-08193 Bellaterra, Spain.

${ }^{7}$ Max-Planck-Institut für Physik, D-80805 Munich, Germany.

${ }^{8}$ Università di Padova and INFN, I-35131 Padova, Italy.

${ }^{9}$ University of Łódź, PL-90236 Lodz, Poland.

${ }^{10}$ Universität Würzburg, D-97074 Würzburg, Germany.

${ }^{11}$ DESY, Deutsches Elektr.-Synchrotron, D-15738 Zeuthen, Germany.

${ }^{12}$ Università di Siena, and INFN Pisa, I-53100 Siena, Italy.

${ }^{13}$ Universitat de Barcelona (ICC/IEEC), E-08028 Barcelona, Spain.

${ }^{14}$ Yerevan Physics Institute, AM-375036 Yerevan, Armenia.

${ }^{15}$ Inst. de Astrofisica de Canarias, E-38200 La Laguna, Tenerife, Spain.

${ }^{16}$ Depto. de Astrofisica, Universidad, E-38206 La Laguna, Tenerife, Spain.

${ }^{17}$ Università di Udine, and INFN Trieste, I-33100 Udine, Italy.

${ }^{18}$ Institut de Cienciès de 1'Espai (IEEC-CSIC), E-08193 Bellaterra, Spain.

${ }^{19}$ Inst. de Astrofisica de Andalucia (CSIC), E-18080 Granada, Spain.

${ }^{20}$ University of California, Davis, CA 95616-8677.

${ }^{21}$ Tuorla Observatory, Turku University, FI-21500 Piikkiö, Finland.

${ }^{22}$ Inst. for Nucl. Research and Nucl. Energy, BG-1784 Sofia, Bulgaria.

${ }^{23}$ Humboldt-Universität zu Berlin, D-12489 Berlin, Germany.

${ }^{24}$ INAF-Osservatorio Astronomico and INFN, I-34143 Trieste, Italy.

${ }^{25}$ ICREA, E-08010 Barcelona, Spain.

${ }^{26}$ Università di Pisa, and INFN Pisa, I-56126 Pisa, Italy.

${ }^{27}$ Now at APC (CNRS), Paris, France.

${ }^{28}$ Now at University of California, Santa Cruz, CA 95064.

${ }^{29}$ Correspondence: J. Rico (jrico@ifae.es), D. F. Torres (dtorres@ieec uab.es). strongest sustained winds among Galactic objects: their terminal velocities may reach the range $v_{\infty}>1000-5000 \mathrm{~km} \mathrm{~s}^{-1}$. Perhaps with the exception of the short-lived luminous blue variables (LBVs), they have the highest known mass-loss rate $\dot{M} \sim 10^{-4}$ to $10^{-5} M_{\odot} \mathrm{yr}^{-1}$ of any stellar type. Thus, colliding winds of massive star binary systems are considered potential sites of nonthermal high-energy photon production, via leptonic and/or hadronic process after acceleration of primary particles in the collision shock (e.g., Eichler \& Usov 1993). This possibility is substantiated by the detection of (nonthermal) synchrotron radio emission from the expected colliding wind location in some binaries (see below). Many models have been proposed to predict $\mathrm{GeV}$ to $\mathrm{TeV}$ emission from these binaries, with different levels of detail (e.g., among recent works see Benaglia et al. 2001; Benaglia \& Romero 2003; Pittard \& Dougherty 2006; Reimer et al. 2006). Conceptually, the process would mimic the cases of LS 5039 (Aharonian et al. 2005b), PSR B1259-63 (Aharonian et al. 2006), or LS I +61 303 (Albert et al. 2006, 2008d, 2008e), particularly if they result in pulsar-driven $\gamma$-ray binaries in which $\gamma$-rays may arise from a shock region produced by the interaction of the winds of the two components. ${ }^{30}$

In a recent paper, the High Energy Stereoscopic System (H.E.S.S.) collaboration reported the discovery of very high energy (VHE) $\gamma$-ray emission coincident with the young stellar

\footnotetext{
${ }^{30} \mathrm{We}$ recall that these binaries have orbital modulated $\mathrm{TeV}$ emission, and are formed by a massive star with a strong wind and a compact object, which only in the case of PSR B1250-63 is known to be a pulsar.
} 


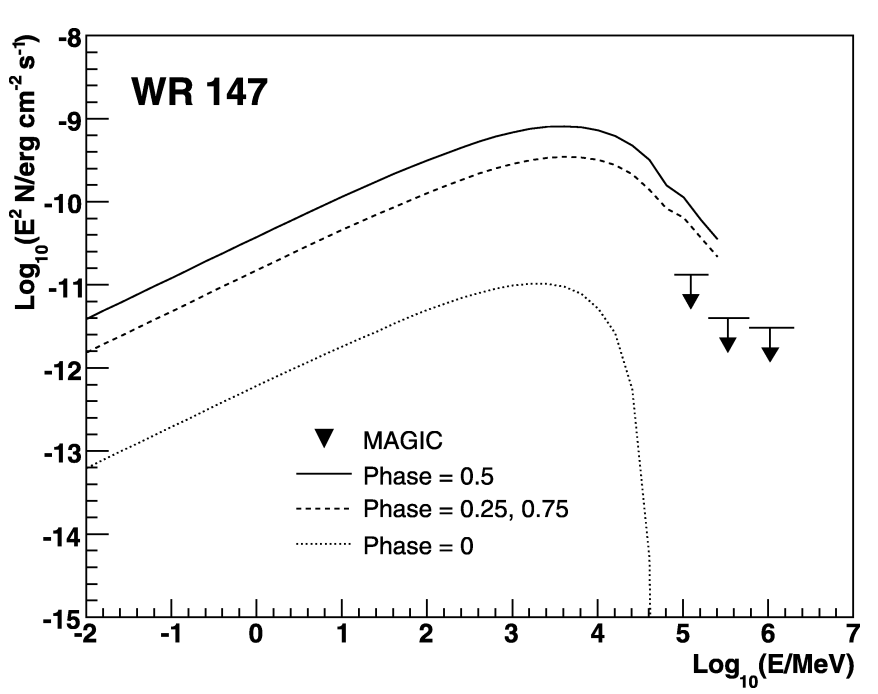

FIG. 1.-Inverse Compton spectra of WR 147 for orbital phases $0,0.25$, 0.5 , and 0.75 , neglecting any eccentricity of the system and assuming $i=$ $90^{\circ}$, from Reimer et al. (2006). $\gamma \gamma$ pair production absorbs not more than $\leq 0.3 \%(>50 \mathrm{GeV})$ and $\leq 18 \%(>100 \mathrm{GeV})$ of the produced flux at orbital phases 0.25 and 0.5 , respectively. No absorption takes place at phase 0 . MAGIC upper limits on this system are marked.

cluster Westerlund 2 (Aharonian et al. 2007). The High Energy Gamma Ray Astronomy (HEGRA) and the Major Atmospheric Gamma Imaging Cerenkov (MAGIC) telescopes detected the source TeV J2032+4130 and suggested a possible connection with the Cygnus OB2 cluster (Aharonian et al. 2002, 2005a; Albert et al. 2008a). Theoretically, the relationship between stellar associations and high-energy emission has been put forward by many authors, since early-type stellar associations have long been proposed as cosmic-ray acceleration sites and also as providers of target material for cosmic-ray interactions (see for instance the recent papers by Parizot et al. 2004; Torres et al. 2004; Bednarek 2005; Domingo-Santamaría \& Torres 2006 and references therein).

In the case of Cygnus OB2, the VHE emission is supposed to occur at a region displaced from the center of the association, where detailed multiwavelength studies revealed an overdensity of hot OB stars, although not WRs (Butt et al. 2003, 2006). In the case of Westerlund 2, the stellar cluster contains at least a dozen early-type $\mathrm{O}$ stars, and two remarkable WR stars, WR 20a and WR 20b. In particular, WR 20a was recently established as a binary (Rauw et al. 2004; Bonanos et al. 2004). Based on the orbital period, the minimum masses were found to be around $83 \pm 5 M_{\odot}$ and $82 \pm 5 M_{\odot}$ for the binary components (Rauw et al. 2005), which certainly qualifies it among the most massive binary systems in our Galaxy. No significant flux variability or orbital periodicity was found in the corresponding data sets, neither for the Cygnus OB2 nor for the Westerlund 2 regions. Unless such an orbital period is detected in future data sets, it would be very difficult or impossible for the current generation of instruments to distinguish whether the radiation observed from these associations is coming from a isolated binary system or rather is generated as a collective effect of the whole cluster. Thus, a direct measurement of single WR binary systems, to explore whether they are able to produce high-energy $\gamma$-ray emission in an isolated condition, is worth pursuing. After briefly motivating the scenario for $\gamma$-ray emission from isolated binary systems, we present the results of MAGIC observations of two such systems.

\section{HIGH-ENERGY $\gamma$-RAYS FROM WR BINARIES: CANDIDATES, OBSERVATIONS, AND RESULTS}

For the present investigation, and apart from other technical considerations like a favorable declination, we looked for candidates with nonthermal emission, indicating the presence of relativistic electrons, and for which the geometry of the colliding wind region is established. The two selected systemsWR 146 and WR 147-have been resolved using the Very Large Array (VLA) and the Multi-Element Radio Linked Interferometer (MERLIN) in two sources each and at least for WR 147, where the most detailed model is currently available, the system was predicted to be a powerful MAGIC source for most of the orbital period (Reimer et al. 2006).

For the angular resolution of the MAGIC telescope $\left(\sim 0.1^{\circ}\right)$ the colliding wind zone will not appear to be spatially resolved, presenting individual colliding wind binary systems as pointsource candidates at the $\gamma$-ray sky. Thus we have searched for point sources in the direction of these two binaries.

\subsection{WR 147}

WR 147 (see, e.g., Setia Gunawan et al. 2001 and references therein), among the closest and brightest systems that show nonthermal radio emission in the $\mathrm{cm}$ band, is composed of a WN8(h) plus a B0.5 V star with a bolometric luminosity of $L_{\text {bol }}=5 \times 10^{4} L_{\odot}$ and effective temperature $T_{\text {eff }}=28,500$ $\mathrm{K}$, i.e., thermal photon energy of about $\epsilon_{T} \approx 6.6 \mathrm{eV}$. At a distance of $650 \mathrm{pc}$ the implied binary separation is estimated to be $417 \mathrm{AU}$. The mass-loss rates $\left(M_{\mathrm{WR}}=2.5 \times 10^{-5} M_{\odot} \mathrm{yr}^{-1}\right.$, $\left.\dot{M}_{\mathrm{OB}}=4 \times 10^{-7} M_{\odot} \mathrm{yr}^{-1}\right)$ and wind velocities $\left(v_{\mathrm{wR}}=950\right.$ $\mathrm{km} \mathrm{s}^{-1}, v_{\text {Ов }}=800 \mathrm{~km} \mathrm{~s}^{-1}$ ) place the stagnation point at $6.6 \times 10^{14} \mathrm{~cm}$. This is in fact in agreement with MERLIN observations, which show a northern nonthermal component and a southern thermal one with a separation of $575 \pm 15$ mas (Churchwell et al. 1992; Williams et al. 1997). This radio morphology and spectrum support a colliding wind scenario (Williams et al. 1997), whose collision region has also been detected by the Chandra X-ray telescope (Pittard et al. 2002).

The nonthermal flux component can be well fitted by a power law with spectral index $\alpha=-0.43$. Neither the eccentricity nor the inclination of the system are known due to the very long orbital period (1350 yr, as derived by Setia Gunawan et al. 2001). Reimer et al. (2006) have provided a detailed modeling of the high-energy $\gamma$-ray emission expected from WR 147. The expected fluxes are shown in Figure 1, together with MAGIC upper limits for which we give further details below.

WR 147 was observed with the MAGIC telescope between 2007 August 11 and September 10, for a total of $30.3 \mathrm{hr}$ of good data (after quality cuts removing bad weather runs). The zenith angle of the observations ranged between $10^{\circ}$ and $30^{\circ}$, being sensitive to gamma rays in the energy range between about $80 \mathrm{GeV}$ and $10 \mathrm{TeV}$. The observations were carried out in the false-source track (wobble) mode (Fomin et al. 1994), with two directions at $24^{\prime}$ distance east-west of the source direction. The analysis of the data was performed with the MAGIC standard analysis chain (Albert et al. 2008c), which combines Hillas image parameters by means of a random forest algorithm (Albert et al. 2008b) for signal/background discrimination and energy estimation. The training of the algorithm is done by means of contemporary data from the Crab Nebula observations and Monte Carlo simulated gamma-ray events. Since 2007 February, MAGIC signal digitization has been upgraded to 2 GSample $\mathrm{s}^{-1}$ flash analog-to-digital converters (FADCs), and timing parameters are used during the data anal- 
TABLE 1

WR 147 OBSERVATION RESUlts

\begin{tabular}{cccr}
\hline \hline $\begin{array}{c}\text { Energy } \\
(\mathrm{GeV})\end{array}$ & $\begin{array}{c}N_{\text {excess }} \\
(\text { events })\end{array}$ & $\begin{array}{c}\text { Significance } \\
(\sigma)\end{array}$ & $\begin{array}{c}\text { Upper Limits } \\
{\left[\text { events }\left(\mathrm{cm}^{-2} \mathrm{~s}^{-1}\right)\right]}\end{array}$ \\
\hline$>80 \ldots \ldots$. & $-196 \pm 175$ & -1.1 & $150\left(1.1 \times 10^{-11}\right)$ \\
$>200 \ldots \ldots$ & $-92 \pm 89$ & -1.0 & $84\left(3.1 \times 10^{-12}\right)$ \\
$>600 \ldots \ldots$ & $-20 \pm 24$ & -0.8 & $28\left(7.3 \times 10^{-13}\right)$ \\
\hline
\end{tabular}

Notes. - From left to right: energy range, number of excess events, statistical significance of the excess (Li \& Ma 1983), and signal upper limit for the different observation nights. Upper limits (Rolke et al. 2005) are 95\% confidence level and are quoted in number of events and (between parentheses) in photon flux units assuming a Crab-like spectrum (Albert et al. 2008c).

ysis (Tescaro et al. 2007). This results in an improvement of the flux sensitivity from $2.5 \%$ to $1.6 \%$ (at a flux peak energy of $280 \mathrm{GeV}$ ) of the Crab Nebula flux in $50 \mathrm{hr}$ of observations.

Searches of gamma rays from WR 147 have been performed for three different energy cuts, namely, above $80 \mathrm{GeV}$, above $200 \mathrm{GeV}$, and above $600 \mathrm{GeV}$. In all cases the number of signal candidate events found are compatible with statistical fluctuations of the expected background. The obtained upper limits are shown in Table 1 and Figure 1, and correspond to 1.5\%, $1.4 \%$, and $1.7 \%$ of the Crab Nebula flux for the three considered energy bins, respectively.

\subsection{WR 146}

WR 146 is a similar system: a WC6+O8 colliding-wind binary system also presenting thermal emission from the stellar winds of the two stars, and bright nonthermal emission from the wind-collision region (e.g., see Dougherty et al. 1996, 2000; O'Connor et al. 2005). The period is estimated to be $\sim 300 \mathrm{yr}$ (Dougherty et al. 1996) and the estimates of the distance to the system differ from 0.75 to $1.7 \mathrm{kpc}$ (see Setia Gunawan et al. 2001 and references therein for other system parameters).

WR 146 is located $\sim 0.7^{\circ}$ away from the unidentified VHE $\gamma$-ray source TeV J2032+4130 and was observed with MAGIC within the observation program devoted to this source (Albert et al. 2008a), albeit with a reduced sensitivity. The total effective exposure, which accounts for the loss of sensitivity of off-axis observations and camera illumination during moonlight observations (Albert et al. 2007), is $44.5 \mathrm{hr}$, obtained between 2005 and 2007. At the MAGIC site, WR 146 culminates at $14^{\circ}$ and the observations were carried out at zenith angles between $14^{\circ}$ and $44^{\circ}$ (see Albert et al. 2008a for details). The data analysis follows the standard MAGIC analysis chain. Since most of the data are acquired with $300 \mathrm{MHz}$ FADCs, image timing parameters are not used in this analysis.

The result of the searches of gamma rays from WR 146 for three different energy cuts (above $80 \mathrm{GeV}$, above $200 \mathrm{GeV}$, and $>600 \mathrm{GeV}$ ) are shown in Table 2. As for the case of WR 147, all measured signal candidates are consistent with background fluctuations and the upper limits (corresponding to
TABLE 2

WR 146 OBSERVATION RESUlts

\begin{tabular}{cccr}
\hline \hline $\begin{array}{c}\text { Energy } \\
(\mathrm{GeV})\end{array}$ & $\begin{array}{c}N_{\text {excess }} \\
(\text { events })\end{array}$ & $\begin{array}{c}\text { Significance } \\
(\sigma)\end{array}$ & $\begin{array}{c}\text { Upper Limits } \\
{\left[\text { events }\left(\mathrm{cm}^{-2} \mathrm{~s}^{-1}\right)\right]}\end{array}$ \\
\hline$>80 \ldots \ldots$ & $264 \pm 97$ & 2.7 & $840\left(3.5 \times 10^{-11}\right)$ \\
$>200 \ldots \ldots$ & $133 \pm 67$ & 2.5 & $487\left(7.7 \times 10^{-12}\right)$ \\
$>600 \ldots \ldots$ & $-21 \pm 26$ & -0.8 & $46\left(5.6 \times 10^{-13}\right)$ \\
\hline
\end{tabular}

Notes. - From left to right: energy range, number of excess events, statistical significance of the excess (Li \& Ma 1983), and signal upper limit for the different observation nights. Upper limits (Rolke et al. 2005) are 95\% confidence level and are quoted in number of events and (between parentheses) in photon flux units assuming a Crab-like spectrum (Albert et al. 2008c).

$5.0 \%, 3.5 \%$, and $1.2 \%$ of the Crab Nebula flux) are presented. At the lower energy bins we see a positive number of excesses at the $\sim 2.5 \sigma$ level. However, the present data are too scarce to establish if this comes from a gamma-ray signal or background fluctuations. Future observations will shed light on this issue.

\section{CONCLUDING REMARKS}

Our search for VHE $\gamma$-ray emission from two archetypical cases of WR binaries produced the first bounds on such systems. These bounds constrain theoretical models (or, assuming correctness of the models, their internal parameters, such as the - unknown —orbital phases of the systems). The establishment of WR binaries as VHE $\gamma$-ray sources is yet pending.

The case for WR 147 as a potential $\gamma$-ray source for MAGIC was theoretically established before, as shown in the corresponding curves of Figure 1 from Reimer et al. (2006) for most of the orbital phases. The validity of this model is baselined on an assumed ensemble of orbital parameters, which are still unknown for this system. For instance, ignorance of its current phase as well as of its eccentricity and inclination makes a direct ruling out of this model impossible, although the presented scenario could nominally survive only for phases close to 0 , defined where the line of sight encounters first the WN8 and then the B0.5 V star. The MAGIC observations show that irrespective of phase, GLAST should see a flux cutoff well within its range of detectability in the tens of $\mathrm{GeV}$ regime, if it is able to detect the stars at all.

We acknowledge A. Reimer for providing us the theoretical curves depicted in Figure 1. We also would like to thank the Instituto de Astrofisica de Canarias for the excellent working conditions at the Observatorio del Roque de los Muchachos in La Palma. The support of the German BMBF and MPG, Italian INFN, and Spanish CICYT is gratefully acknowledged. This work was also supported by ETH research grant TH 34/043, by the Polish MNiSzW grant N N203 390834, and by the YIP of the Helmholtz Gemeinschaft.

\section{REFERENCES}

Aharonian, F., et al. 2002, A\&A, 393, L37

2005a, A\&A, 431, 197

2005b, A\&A, 442, 1

2006, A\&A, 460, 743

2007, A\&A, 467, 1075

Albert, J., et al. 2006, Science, 312, 1771

—. 2007, preprint (astro-ph/0702475)

2008a, ApJ, 675, L25

2008b, Nucl. Instrum. Methods Phys. Res., A588, 424

2008c, ApJ, 674, 1037
Albert, J., et al. 2008d, ApJ, 684, 1351

2008e, ApJ, submitted (arXiv:0806.1865)

Bednarek, W. 2005, MNRAS, 363, L46

Benaglia, P., \& Romero, G. E. 2003, A\&A, 399, 1121

Benaglia, P., Romero, G. E., Stevens, I., \& Torres, D. F. 2001, A\&A, 366, 605

Bonanos, A. Z., et al. 2004, ApJ, 611, L33

Butt, Y., et al. 2003, ApJ, 597, 494

2006, ApJ, 643, 238

Churchwell, E., et al. 1992, ApJ, 393, 329 
Domingo-Santamaría, E., \& Torres, D. F. 2006, A\&A, 448, 613

Dougherty, S. M., Williams, P. M., \& Pollaco, D. L. 2000, MNRAS, 316, 143

Dougherty, S. M., et al. 1996, MNRAS, 280, 963

Eichler, D., \& Usov, H. 1993, ApJ, 402, 271

Fomin, V. P., Stepanian, A. A., Lamb, R. C., Lewis, D. A., Punch, M., \& Weekes, T. C. 1994, Astropart. Phys., 2, 137

Li, T.-P., \& Ma, Y.-Q. 1983, ApJ, 272, 317

O'Connor, E., Dougherty, S., Pittard, J., \& Williams, P. 2005, JRASC, 99, 142

Parizot, E., et al. 2004, A\&A, 424, 727

Pittard, J. M., \& Dougherty, S. M. 2006, MNRAS, 372, 801

Pittard, J. M., et al. 2002, A\&A, 388, 335
Rauw, G., et al. 2004, A\&A, 420, L9 2005, A\&A, 432, 985

Reimer, A., Pohl, M., \& Reimer, O. 2006, ApJ, 644, 1118

Rolke, W. A., López, A. M., \& Conrad, J. 2005, Nucl. Instrum. Methods Phys. Res., A551, 493

Setia Gunawan, D. Y. A., de Bruyn, A. G., van der Hucht, K. A., \& Williams, P. M. 2001, A\&A, 368, 484

Tescaro, D., et al. 2007, preprint (arXiv:0709.1410)

Torres, D. F., Domingo-Santamaría, E., \& Romero, G. E. 2004, ApJ, 601, L75

Williams, P. M., et al. 1997, MNRAS, 289, 10

Note added in proof.- - Recently we came across a paper by A. C. Rovero et al. (in IAU Symp. 163, Wolf-Rayet Stars: Binaries, Colliding Winds, Evolution, ed. K. A. van der Hucht \& P. M. Williams [Dordrecht: Kluwer, 1995]), where upper limits to WR stars are presented. These are far less restrictive than the ones shown here, having been made with the first generation of IACT arrays. 